\title{
Inelasticity in p-nucleus collisions and its application to high energy cosmic-ray cascades
}

\author{
G. M. Frichter, T. K. Gaisser and T. Stanev \\ Bartol Research Institute, University of Delaware \\ Newark, Delaware 19716, USA
}

(August 17, 2021)

We employ a simple multiple scattering model to investigate the inclusive reaction $\mathrm{p}+\mathrm{A} \rightarrow \mathrm{p}+\mathrm{X}$ for projectile momenta in the $100-200 \mathrm{GeV} / \mathrm{c}$ range. We find that data are consistent with a class of interaction models in which the stopping power of nuclei is rather low. We discuss extrapolation to ultra-high energy and the application to interpretation of cosmic-ray air showers at energies up to $10^{20} \mathrm{eV}$.

PACS numbers: 95.55.Vj, 96.40.De, 96.40.Pq, 25.40.Ep, 13.85. Tp, $13.85 . \mathrm{Ni}, 13.60 . \mathrm{Hb}$

\section{INTRODUCTION}

To explore the cosmic-ray spectrum beyond about $10^{15} \mathrm{eV}$ requires ground-based experiments with large effective area and long exposure times to overcome the increasingly low flux implied by the steeply falling energy spectrum. Such air shower experiments cannot observe the primary particle directly but can only sample the cascade it generates in the atmosphere. For this reason, obtaining results of astrophysical interest, such as the relative fraction of different types of nuclei or the fraction of gamma-rays, requires extensive Monte Carlo simulation to model the cascades and interpret the measurements.

A recurring problem is that uncertainties in the input to the calculations introduce corresponding ambiguities in the interpretations of the experiments. A major, and to some extent unavoidable, source of uncertainty is the modelling of the hadronic interactions at energies well beyond those explored at accelerators. In the extreme case, protons with energies near the Greisen-Zatsepin-Kuz'min (GZK) cutoff [1] at $\sim 5 \times 10^{19} \mathrm{eV}$ correspond to center-of mass energies more than two orders of magnitude beyond the highest energy hitherto available at hadron colliders. There are uncertainties also in the region of the "knee" of the spectrum around $5 \times 10^{15} \mathrm{eV}$ even though this is approximately equivalent to the center of mass energy of the Fermilab collider. There are two reasons that significant uncertainties remain even at this relatively low energy. One is that interactions in the atmosphere involve nuclear targets, and in some cases nuclear projectiles as well. The other is that it is the forward fragmentation region of the collision - largely unexplored by collider detectors - that primarily determines the rate of energy deposition that generates the core of the atmospheric cascades.

The most global properties of minimum-bias hadronic interactions determine the development of air showers.
These include the cross section and the inelasticity. The $\bar{p} p$ cross section is directly measured up to $\sqrt{s} \approx 2 \mathrm{TeV}$, and its extension to higher energy can be obtained by extrapolation of fits based on Regge theory. [2] There is less agreement on how to extrapolate inelasticity and related quantities that determine the rate at which energy is deposited in the atmosphere via electromagnetic subshowers. We focus on inelasticity in this paper.

We are motivated to study this problem now because of intense experimental activity and ambitious new proposals aimed at the highest energy cosmic rays [3] 7] as well as highly instrumented hybrid arrays aimed at discovering the sources of cosmic-rays that give rise to the knee feature in the spectrum, for example 8 12]. There is a corresponding interest in simulations as illustrated by the systematic comparison of several codes undertaken by the group at Karlsruhe. 13] By installing several hadronic event generators into the same cascade code, they have isolated differences due to the input physics of the inteaction models from possible technical differences in how the cascades are followed. The latter in principle should not be sources of uncertainty in any case, being determined by well-known physics such as energy-loss by ionization, pair-production, bremsstrahlung, etc.

Qualitatively, the inelasticity of a hadronic interaction is the fraction of the beam energy not carried off by the fragment of the incoming particle. This fraction of the energy is then available for particle production, including neutral pions which transfer energy from the hadronic core of the shower into electromagnetic subshowers. Inelasticity is just one moment of one of the inclusive distributions, but it is arguably the most significant for cascade development (next to the inelastic cross section itself) because it determines the rate at which the initial energy of the cascade dissipates.

For $p p$ collisions there is a precise experimental definition that involves the inclusive cross sections for production of protons, neutrons and their antiparticles. Defining

$$
\frac{d \sigma_{N}}{d^{3} p}=\left[\frac{d \sigma_{p}}{d^{3} p}+\frac{d \sigma_{n}}{d^{3} p}-\frac{d \sigma_{\bar{p}}}{d^{3} p}-\frac{d \sigma_{\bar{n}}}{d^{3} p}\right]
$$

we have

$$
\int \frac{d \sigma_{N}}{d^{3} p} d^{3} p=2 \sigma_{\text {inel }}
$$

(because there are two nucleons in the initial state) and 


$$
\int E \times \frac{d \sigma_{N}}{d^{3} p} d^{3} p=\sqrt{s} \times K_{\mathrm{el}} .
$$

Here $K_{\mathrm{el}}$ is the elasticity, and the inelasticity is defined as

$$
I=\left[1-K_{\mathrm{el}}\right] .
$$

For pion-initiated interactions, a precise definition of elasticity requires a model because of the essential ambiguity between produced and fragment pions. At high energy the elasticity defined in Eq. 3 is approximated by the integral over the leading nucleon in the lab frame, which is the definition we use in the remainder of this paper.

For p-nucleus collisions we follow the work of Refs. 114,15 and consider partial inelasticities in the framework of a Glauber multiple scattering formalism [16]. The elasticity is given by

$$
K_{\mathrm{el}}=\langle E\rangle=\sum P_{\nu}\langle E\rangle_{\nu},
$$

where

$$
P_{\nu}=\frac{\sigma_{\nu}^{\mathrm{pA}}}{\sigma_{\text {inel }}^{\mathrm{pA}}}
$$

is the probability for exactly $\nu$ wounded nucleons in a target of mass $A$ and $\langle E\rangle_{\nu}$ is the mean energy of the leading nucleon in collisions with exactly $\nu$ wounded nucleons. The partial inelasticity coefficient is defined by the relation

$$
\langle E\rangle_{\nu}=\left(1-I_{\nu}\right) \times\langle E\rangle_{\nu-1} .
$$

The total inelastic hadron-nucleus cross section is $\sigma_{\text {inel }}^{\mathrm{pA}}=\sum \sigma_{\nu}^{\mathrm{pA}}$, and the mean number of wounded nucleons is

$$
\langle\nu\rangle=A \frac{\sigma_{\mathrm{pp}}}{\sigma_{\mathrm{pA}}} .
$$

We calculate both $\sigma_{\text {inel }}^{\mathrm{pA}}$ and the partial cross sections $\sigma_{\nu}^{\mathrm{pA}}$ from the cross sections for $p p$ scattering and the nuclear profiles as described in Ref. 117.

As emphasized in Ref. [15], there is no basis for a naive interpretation of Eq. / because fast fragment(s) of the projectile do not reach an asymptotic physical state until well outside the nucleus. The strategy is to assume that $I_{1}$ is determined by $p p$ scattering and to treat the remaining partial inelasticities $(\nu>1)$ as free parameters constrained by fitting $p$-nucleus data within the framework of the model. Our method and conclusions are similar to those of Ref. [15], although we have improved on their analysis by using a larger data sample, by treating neutrons and protons separately and by considering effects of diffraction.

The paper is organized in the following way. Section 2 describes in detail the multiple scattering model we use for the description of proton-nucleus interactions. Section 3 introduces the fits to $p p$ data that we need for the definition of $I_{1}$ and other parameters for the case $\nu=1$. In section 4 we give the fits to p-nucleus data and the resulting values for $I_{\nu>1}$. Section 5 contains a discussion of the results in the context of models in current use for calculations of cosmic ray cascades at extremely high energies. and examples of estimated inelasticities in proton air collisions at very high energy. Section 6 gives a summary of the results and conclusions.

\section{THE MULTIPLE SCATTERING MODEL}

The outgoing nucleon in the reaction $\mathrm{p}+\mathrm{A} \rightarrow \mathrm{N}+\mathrm{X}$ ( $\mathrm{N}$ being either neutron or proton) can be specified by its transverse and longitudinal momenta, $p_{\mathrm{t}}$ and $x=p_{\mathrm{l}} / p_{o}$ where $p_{o}$ is the incident proton momentum. We model the differential cross section for this process as a sum over final state distributions corresponding to definite numbers of wounded nucleons, $\nu$,

$$
\frac{d^{3} \sigma^{\mathrm{p}+\mathrm{A} \rightarrow \mathrm{N}+\mathrm{X}}}{d p_{\mathrm{t}}^{2} d x}=\sum_{\nu=1}^{\mathrm{A}} \sigma_{\nu}^{\mathrm{pA}} M_{\nu}^{\mathrm{N}}(x) \frac{b_{\nu}^{\mathrm{N}}(x)^{2}}{2 \pi} e^{-b_{\nu}^{\mathrm{N}}(x) p_{\mathrm{t}}} .
$$

The transverse momentum distributions are assumed to be described sufficiently by an exponential form for fixed values of $x$ and are specified by the slope functions $b_{\nu}^{\mathrm{N}}(x)$. Longitudinal momentum distributions for final state nucleons are contained in the functions $M_{\nu}^{\mathrm{N}}(x)$ which are normalized as

$$
\int_{0}^{1} d x M_{\nu}^{\mathrm{p}}(x)=n_{\nu}^{\mathrm{p}},
$$

and

$$
\int_{0}^{1} d x M_{\nu}^{\mathrm{n}}(x)=n_{\nu}^{\mathrm{n}}
$$

with

$$
n_{\nu}^{\mathrm{p}}+n_{\nu}^{\mathrm{n}}=1 .
$$

The numbers $n_{\nu}^{\mathrm{N}}$ express the outgoing nucleon multiplicities for each number of wounded target nucleons. So $M_{\nu}^{\mathrm{p}}$ and $M_{\nu}^{\mathrm{n}}$ give the $x$-distributions and relative numbers of protons and neutrons after $\nu$ collisions. Eq. 12 expresses the fact that our analysis follows only the forward outgoing nucleon. When Eq. 9 is integrated over all final nucleon momenta one recovers the inelastic pA cross section times the mean nucleon multiplicity as expected.

Experimental data on the processes $\mathrm{p}+\mathrm{p} \rightarrow \mathrm{p}+\mathrm{X}$ and $\mathrm{p}+\mathrm{p} \rightarrow \mathrm{n}+\mathrm{X}$ may be used to fix the $\left(x, p_{\mathrm{t}}\right)$ distributions and the nucleon multiplicities for $\nu=1$. For larger numbers of wounded nucleons we employ the iterative scheme discussed above (Eq. 7), which is similar in spirit 
to that used by Hwa 14 and also Hufner and Klar. 15 The longitudinal distributions are related by

$$
\begin{aligned}
M_{\nu}^{\mathrm{p}}(x)= & \int_{x}^{1} \frac{d y}{y}\left[S_{\nu-1}^{+}(y) \beta_{\nu-1} M_{\nu-1}^{\mathrm{p}}(x / y)\right. \\
& \left.+S_{\nu-1}^{-}(y)\left(1-\beta_{\nu-1}\right) M_{\nu-1}^{\mathrm{n}}(x / y)\right]
\end{aligned}
$$

and

$$
\begin{aligned}
M_{\nu}^{\mathrm{n}}(x)= & \int_{x}^{1} \frac{d y}{y}\left[S_{\nu-1}^{+}(y) \beta_{\nu-1} M_{\nu-1}^{\mathrm{n}}(x / y)\right. \\
& \left.+S_{\nu-1}^{-}(y)\left(1-\beta_{\nu-1}\right) M_{\nu-1}^{\mathrm{p}}(x / y)\right] .
\end{aligned}
$$

The superscripts + and - above describe interactions which preserve and change the projectile isospin respectively, with the parameters $\beta$ specifying the fraction of isospin preserving reactions. After $\nu-1$ collisions, a nucleon having longitudinal momentum fraction $x / y$ has probability $S_{\nu-1}^{+,-}(y)$ to transition to a state having momentum fraction $x$.

For the case of an incident proton beam, we can take the nucleon distributions after zero collisions as boundary conditions,

$$
M_{0}^{\mathrm{p}}(x)=\delta(1-x)
$$

and

$$
M_{0}^{\mathrm{n}}(x)=0
$$

and require that Eqs. 13 and 14 reproduce the experimentally determined distributions $M_{1}^{\mathrm{p}}(x)$ and $M_{1}^{\mathrm{n}}(x)$. One immediately finds

$$
\begin{aligned}
& S_{0}^{+}(y)=\frac{M_{1}^{\mathrm{p}}(y)}{\int_{0}^{1} d y M_{1}^{\mathrm{p}}(y)}, \\
& S_{0}^{-}(y)=\frac{M_{1}^{\mathrm{n}}(y)}{\int_{0}^{1} d y M_{1}^{\mathrm{n}}(y)},
\end{aligned}
$$

and $\beta_{0}=n_{1}^{\mathrm{p}}$.

Generalizing this result to allow for different inelasticities upon subsequent collisions, we adopt the power law form with a set of adjustable parameters, $\alpha_{\nu}$, to be determined by fits to $\mathrm{pA}$ data.

$$
S_{\nu}^{+,-}(y)=\frac{y^{\alpha_{\nu}} M_{1}^{\mathrm{p}, \mathrm{n}}(y)}{\int_{0}^{1} d y y^{\alpha_{\nu}} M_{1}^{\mathrm{p}, \mathrm{n}}(y)}
$$

When $\alpha_{\nu}=0$ we recover a 'naive' multiple scattering model in which all collisions proceed equally like isolated pp events. With $\alpha_{\nu}>0$ contributions from non-leading collisions $(\nu>1)$ are harder than the initial collision. We will show that the existing data on $\mathrm{pA}$ interactions strongly supports leading baryon spectra that are significantly harder for $\nu>1$ than for $\nu=1$.

In order to define partial inelasticity within our formalism we calculate the mean value of $x$ after $\nu$ collisions, $<x>>_{\nu}^{\mathrm{p}, \mathrm{n}}=\int_{0}^{1} d x x M_{\nu}^{\mathrm{p}, \mathrm{n}}(x)$ and relate it to $\left\langle x>_{\nu-1}^{\mathrm{p}, \mathrm{n}}\right.$ with the ratio giving the elasticity coefficient for the $\nu^{\text {th }}$ collision (or one minus the inelasticity coefficient). Integrating Eqs. 13 and 14 in this manner one arrives at the relation,

$$
\begin{aligned}
& {\left[n_{\nu}^{\mathrm{p}}<x>_{\nu}^{\mathrm{p}}+n_{\nu}^{\mathrm{n}}<x>_{\nu}^{\mathrm{n}}\right]=\left(1-I_{\nu}\right)} \\
& \times\left[n_{\nu-1}^{\mathrm{p}}<x>_{\nu-1}^{\mathrm{p}}+n_{\nu-1}^{\mathrm{n}}<x>_{\nu-1}^{\mathrm{n}}\right]
\end{aligned}
$$

where the mean inelasticity is

$$
\begin{aligned}
I_{\nu}= & 1-\beta_{\nu-1} \int_{0}^{1} d y y S_{\nu-1}^{+}(y) \\
& -\left(1-\beta_{\nu-1}\right) \int_{0}^{1} d y y S_{\nu-1}^{-}(y) .
\end{aligned}
$$

\section{FITS TO P + P DATA}

In the current model, the forms determined for $M_{1}^{\mathrm{p}, \mathrm{n}}(x)$ and $b_{1}^{\mathrm{p}, \mathrm{n}}(x)$ by fitting the available $\mathrm{p}+\mathrm{p} \rightarrow \mathrm{p}+\mathrm{X}$ and $\mathrm{p}+\mathrm{p} \rightarrow \mathrm{n}+\mathrm{X}$ data can be thought of as a set of initial conditions that play a crucial role in what we will eventually infer about leading baryon inelasticity from the p-nucleus data. This is true primarily because the $\nu=1$ term of Eq. 9 represents $20-30 \%$ of the cross section even for the heaviest nuclei, and secondarily due to the connection between $M_{\nu>1}^{\mathrm{N}}(x)$ and $M_{1}^{\mathrm{N}}(x)$ prescribed by Eqs. 13 and 14. Our fitting procedure is straightforward; we use Eq. 9 with $\nu=1$ and expand $M_{1}^{\mathrm{p}, \mathrm{n}}(x)$, and $b_{1}^{\mathrm{p}, \mathrm{n}}(x)$ each in a finite Taylor series. The coefficients are then adjusted to minimize the $\chi^{2}$ per degree of freedom when compared to data.

Figure (11) shows the differential cross section for the process $\mathrm{p}+\mathrm{p} \rightarrow \mathrm{p}+\mathrm{X}$ as a function of longitudinal momentum fraction, $x$, for several values of the transverse momentum between 0.3 and $1.0 \mathrm{GeV} / \mathrm{c}$. The data are for beam momenta of 100 and $175 \mathrm{GeV} / \mathrm{c}$ taken from references 19] and 20] and the curves represent our best fit.

In Fig. (2) we plot the differential cross section for $\mathrm{p}+\mathrm{p} \rightarrow \mathrm{n}+\mathrm{X}$ as a function of $x$ for four transverse momentum bins from 0.15 to $0.75 \mathrm{GeV} / \mathrm{c}$. The neutron data correspond to lab system beam momenta of 282 , 500, 1060, and $1500 \mathrm{GeV} / \mathrm{c}$ from reference [21], and the curves show our best fit. Our fits suggest $b_{1}^{\mathrm{n}}(x) \approx b_{1}^{\mathrm{p}}(x)$, so we will not distinguish between them in the remainder of our discussion.

In fitting the distributions of protons we have separated the single diffractive and non-single diffractive components, so that $M_{\nu}^{\mathrm{p}}(x)=M_{\nu}^{\mathrm{p}, \mathrm{sd}}(x)+M_{\nu}^{\mathrm{p}}$,nsd $(x)$. The forward diffractive component (target dissociation) represents approximately $10 \%$ of the inelastic $p p$ cross section. For its $x$-dependence we use the functional form $(1-x)^{-1}$ [18, with a kinematical cutoff near $x=1$. With forward diffraction fixed, we then fit $M_{\nu}^{\mathrm{p}, \mathrm{nsd}}(x)$ by 


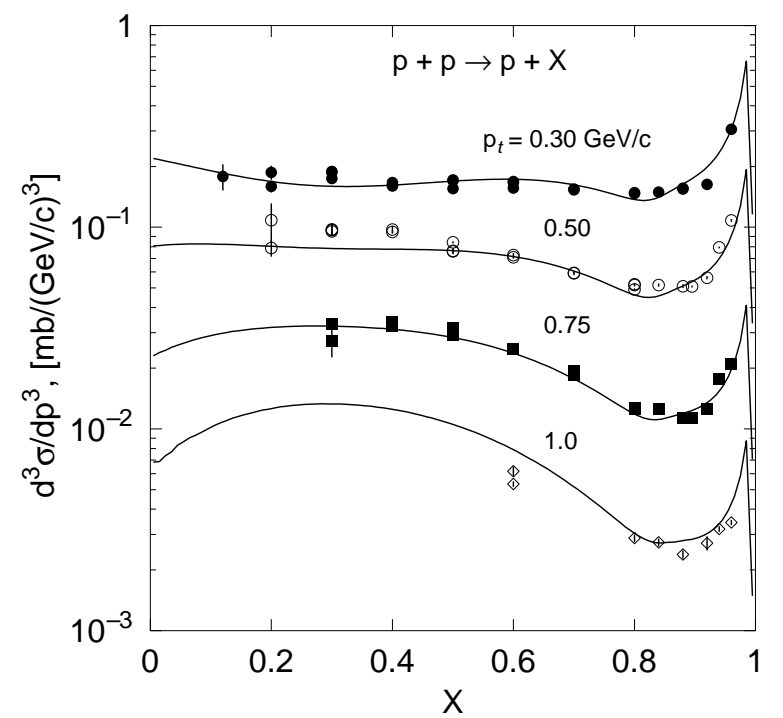

FIG. 1. Differential cross section for $\mathrm{p}+\mathrm{p} \rightarrow \mathrm{p}+\mathrm{X}$ plotted versus momentum fraction $x$ for several transverse momentum bins. The data are for beam momenta of 100 and $175 \mathrm{GeV} / \mathrm{c}$ from references 19) and 20). The curves show our best fit to the data.

the $\chi^{2}$ procedure described above. In our analysis of $p A$ data below, we make the approximation of including the diffractive component only in the case $\nu=1$. This means that diffractive contributions to $\mathrm{pA}$ spectra are confined in our approach primarily to the region $x \geq 0.85$.

The functions $M_{1}^{\mathrm{p} \text {,nsd }}, M_{1}^{\mathrm{n}}$, and $b_{1}$ obtained from the best fits are shown in Fig. (3) along with the diffractive component $M_{1}^{\mathrm{p}, \mathrm{sd}}$ to complete the picture. We find for non-single diffractive protons $\langle x\rangle_{1}^{\mathrm{p} \text {,nsd }}=0.44$ and for neutrons $\left\langle x>_{1}^{\mathrm{n}}=0.26\right.$. The proton and neutron multiplicities derived from our fits (including single diffraction) are $n_{1}^{\mathrm{p}}=0.62$ and $n_{1}^{\mathrm{n}}=0.27$. This should be compared with a proton/neutron ratio of $2: 1$ in a naive constituent quark picture of non-diffractive collisions. Given the overall normalization uncertainties in the pp data (estimated to be $\sim 20 \%$ ), the fitted results are remarkably close. For the calculation of nuclear processes the distributions are normalized so that there is exactly one nucleon (proton or neutron) propagating through the nucleus. (For the first wounded nucleon only the normalized distribution includes the diffractive component.)

We note from our fits that final state neutrons appear to be significantly softer than their non-diffractive proton counterparts, although the uncertainties in the data for $p p \rightarrow n+\mathrm{X}$ are larger than for production of protons. This difference in momentum distribution for neutrons and protons can have two important consequences. First, in Eqs. 13 and 14 there is a 'mixing' of neutron and proton spectra controlled by the parameters $\beta_{\nu>0}$. It is easy to see that the amount of this mixing can af-

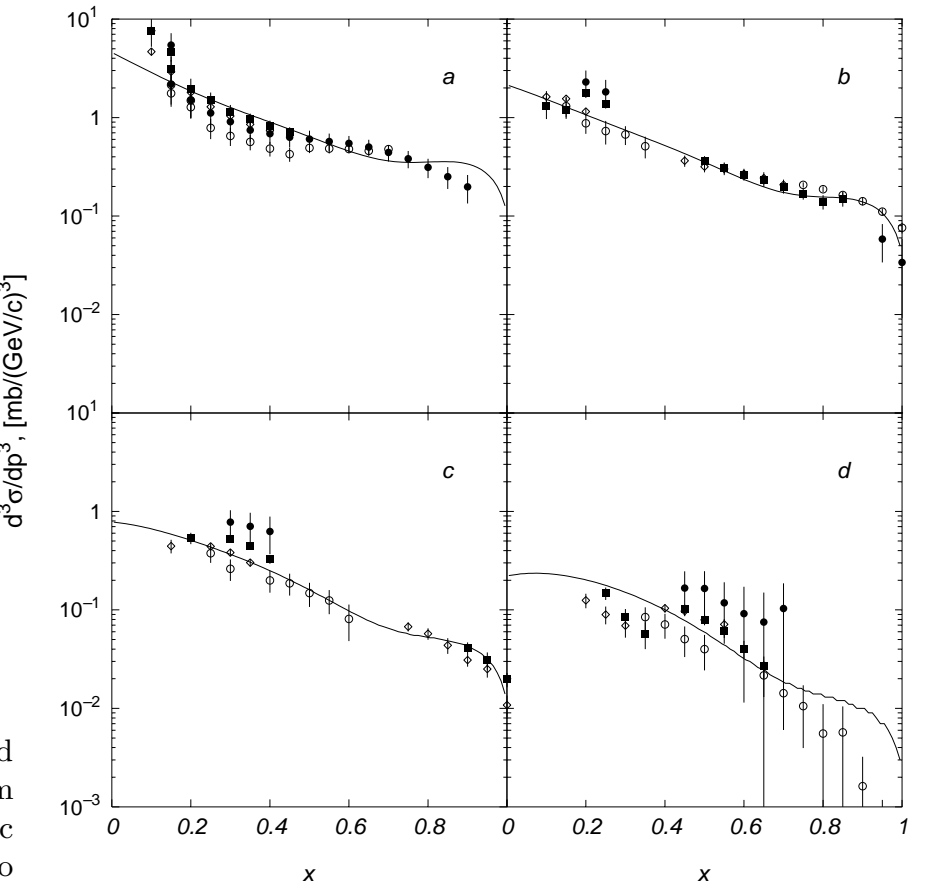

FIG. 2. Differential cross section for $\mathrm{p}+\mathrm{p} \rightarrow \mathrm{n}+\mathrm{X}$ plotted versus momentum fraction $x$ for four transverse momentum values: a) $0.15 \mathrm{GeV} / \mathrm{c}$; b) $0.30 \mathrm{GeV} / \mathrm{c}$; c) $0.50 \mathrm{GeV} / \mathrm{c}$; and d) $0.75 \mathrm{GeV} / \mathrm{c}$. These ISR data correspond to laboratory system beam momenta of 282 (filled circles), 500 (open circles), 1060 (filled squares), and $1500 \mathrm{GeV} / \mathrm{c}$ (open diamonds) from Ref. 21. We have used fits of the form of Eq. $9(\nu=1)$ to interpolate the data to selected values of $x$ and $p_{T}$. The curves show our best fit to the data. 


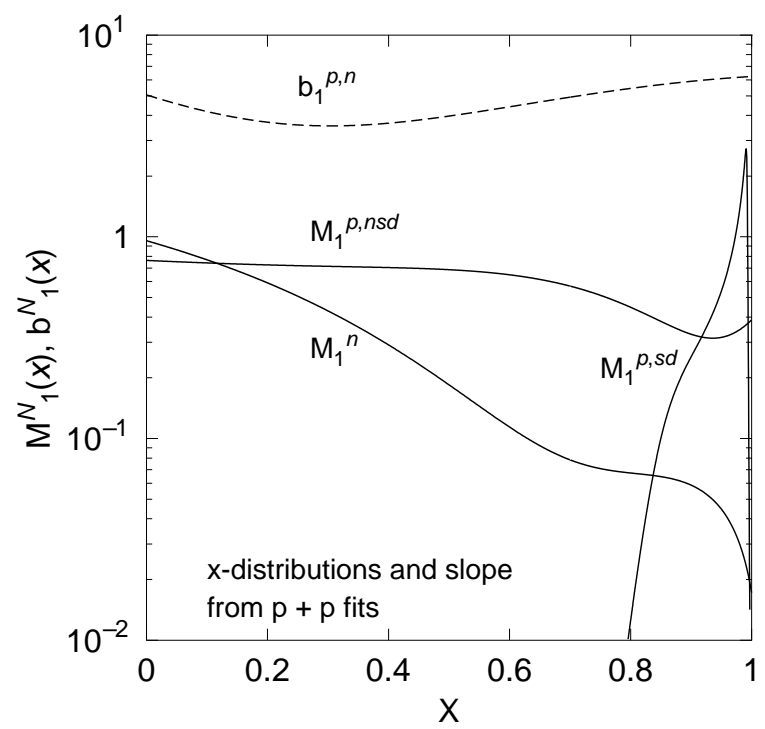

FIG. 3. Probability distributions for final state protons, $M_{1}^{\mathrm{p}, \text { nsd }}(x), M_{1}^{\mathrm{p}, \mathrm{sd}}(x)$, and neutrons, $M_{1}^{\mathrm{n}}(x)$, extracted from fits to $\mathrm{p}+\mathrm{p}$ data. Also shown is the slope function, $b_{1}^{\mathrm{N}}(x)$. The mean momentum fractions and multiplicities are quoted in the text.

fect fits to p-nucleus proton spectra if the input $(\nu=1)$ spectra differ. We will examine this in the next section. Second, because the inital neutrons are softer, the leading nucleon inelasticity relevant to high energy cascade simulation may be less than one predicted on the basis of proton data alone together with the assumption that the inclusive distribution of neutrons is similar to that of non-diffractive protons.

\section{FITS TO P + NUCLEUS DATA: INELASTICITY}

The number of terms to keep in Eq. 9 can be guided by considering the Glauber probabilities $\sigma_{\nu}^{\mathrm{pA}} / \sigma_{\text {inel }}^{\mathrm{pA}}$ for heavy target nuclei (mass $\sim 200$ ). One finds that roughly $90 \%$ of the cross section is obtained by the first five terms and $99 \%$ by the first eleven. We have terminated the sum at $\nu=12$.

Twelve terms results in a large number of parameters to be fit unless some additional assumptions are made. Our approach is to treat all interactions subsequent to the initial one on the same footing. This means that $\alpha_{\nu-1}, \beta_{\nu-1}$, and the functions $b_{\nu}(x)$ have the same value for $\nu>1$. Indeed we have checked that relaxing this constraint has no substantial impact on the results. Only marginally better fits are obtained if, for example, we allow a different $\alpha$ for each value of $\nu$.

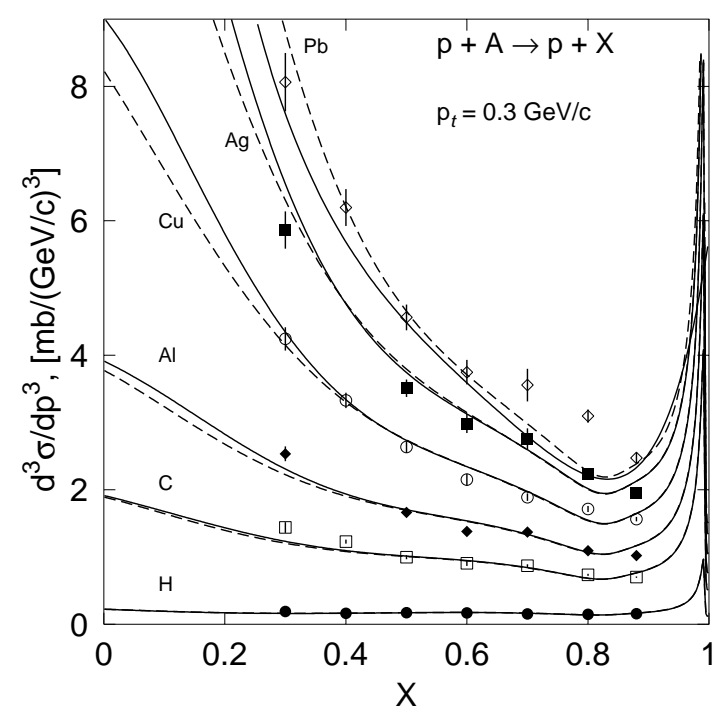

FIG. 4. Differential cross section for the process $\mathrm{p}+\mathrm{A} \rightarrow \mathrm{p}+\mathrm{X}$ plotted versus momentum fraction $x$ at fixed $p_{\mathrm{t}}=0.3 \mathrm{GeV} / \mathrm{c}$ for $100 \mathrm{GeV} / \mathrm{c}$ protons on targets ranging from hydrogen to lead. The data are from reference 20). The dashed and solid curves show our best fits with $\beta=2 / 3$ and $\beta=1$ respectively.

We give the slope functions the simple quadratic form $b_{\nu>1}(x)=a+b x+c x^{2}$ and have checked that higher order terms do not substantially improve the fits to data. These three parameters together with $\alpha_{\nu \geq 1}$ and $\beta_{\nu \geq 1}$ give a total of five free parameters for fitting the p-nucleus data.

We have examined two distinct cases for the value of $\beta_{\nu \geq 1}$ based on different extreme pictures for the nucleon propagation through the nucleus. The first can be thought of as the naive case in which all interactions proceede identically; that is, the probability for isospin preserving reactions at each step is just equal to the proton multiplicity observed in $\mathrm{p}+\mathrm{p}$ reactions, $\beta_{\nu \geq 1}=n_{1}^{\mathrm{p}}=2 / 3$. Note that in this case the probability that the leading nucleon is a proton quickly approaches $1 / 2$ with increasing $\nu\left(14 / 27\right.$ for $\nu=3$ and is $\left.\frac{3^{5}-1}{2 \times 3^{\nu}}\right)$. The opposite extreme is that the isospin of the leading nucleon is determined solely at the first interaction. This second case corresponds to $\beta_{\nu>1}=1$. We will show that these two pictures lead to somewhat different conclusions for the inelasticity of non-leading interactions required to fit the data.

The data we use to study inelasticity are inclusive proton spectra from $\mathrm{p}+$ nucleus reactions by Barton et al. 20] and Bailey et al. 22] with beam energies of 100 and $120 \mathrm{GeV}$ respectively. The $100 \mathrm{GeV}$ data were collected for $\mathrm{C}, \mathrm{Al}, \mathrm{Cu}, \mathrm{Ag}$, and $\mathrm{Pb}$ targets for two transverse momentum bins of 0.3 and $0.5 \mathrm{GeV} / \mathrm{c}$. The $120 \mathrm{GeV}$ 


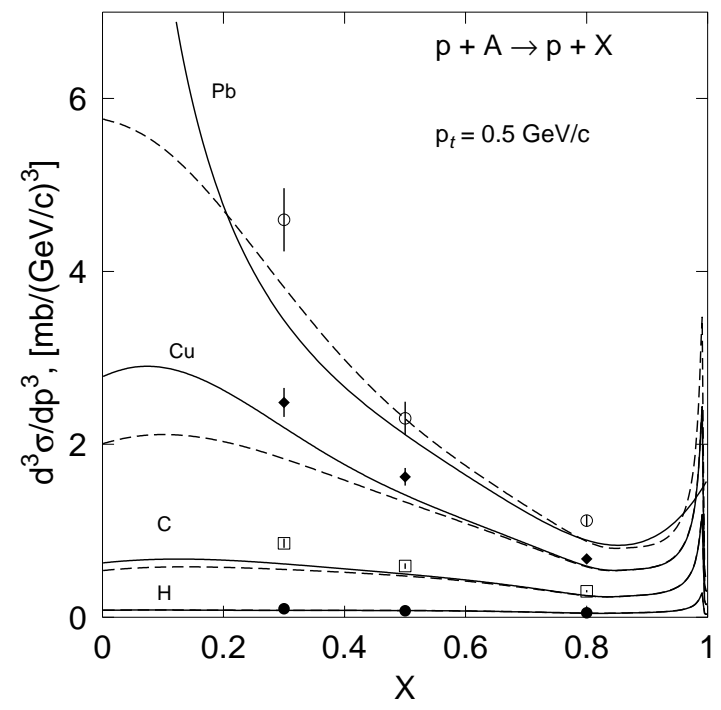

FIG. 5. Same as Fig. (4) but with $p_{\mathrm{t}}=0.5 \mathrm{GeV} / \mathrm{c}$.

data were reported summed over transverse momenta for $\mathrm{Be}, \mathrm{Cu}, \mathrm{Ag}, \mathrm{W}$, and $\mathrm{U}$ targets. These data along with our best fits are shown in Figs. (4), (5), and (6). The dashed and solid curves in these figures correspond to the $\beta=2 / 3$ and $\beta=1$ scenarios respectively.

In Fig. (7) we show the $\chi^{2}$ per degree of freedom statistic of these fits plotted as a function of the inelasticity of non-leading interactions as determined according to Eq. 21. The $\beta=2 / 3$ and $\beta=1$ scenarios yield their best fits for $I=.14$ and .18 respectively. The $\beta=1$ case offers a somewhat better overall fit to the data. It is clear that a naive multiple scattering picture which corresponds to the case of $\beta=2 / 3$ and $I=.5$ is certainly not supported in the present analysis.

\section{IMPLICATIONS FOR CASCADES}

Most hadronic models currently in use for calculation of air showers at high energy fall into one of two categories. One group 23 26 is based on the dual parton model (DPM) 27 or the related Quark-Gluon String model (QGS) 28]. Another approach is to use some variation of statistical or thermodynamical ideas [29,30], producing particles via clusters or fireballs, but constrained to agree with the observed persistence of some high-energy fragments of the projectiles. There are several examples of this type of model, for example Refs. 31 33. Here we focus on one particular model 34] that has been used recently to reevaluate implications of the Fly's Eye measurements for cosmic-ray composition

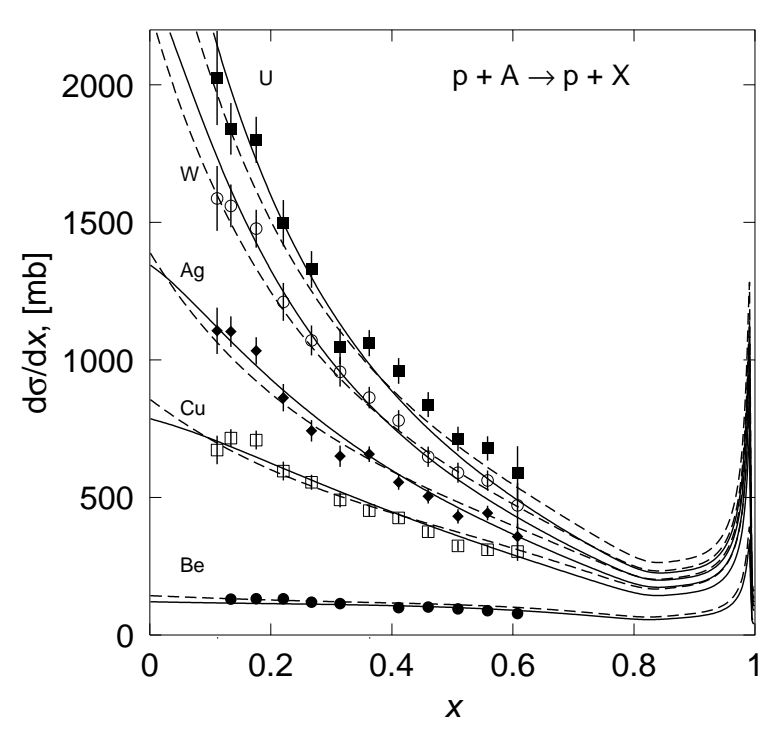

FIG. 6. Differential cross section $d \sigma / d x$ for the process $\mathrm{p}+\mathrm{A} \rightarrow \mathrm{p}+\mathrm{X}$ plotted versus momentum fraction $x$ for 120 $\mathrm{GeV} / \mathrm{c}$ protons on targets ranging from Beryllium to Tungsten. The data are from reference 22). The dashed and solid curves show our best fit with $\beta=2 / 3$ and $\beta=1$ respectively.

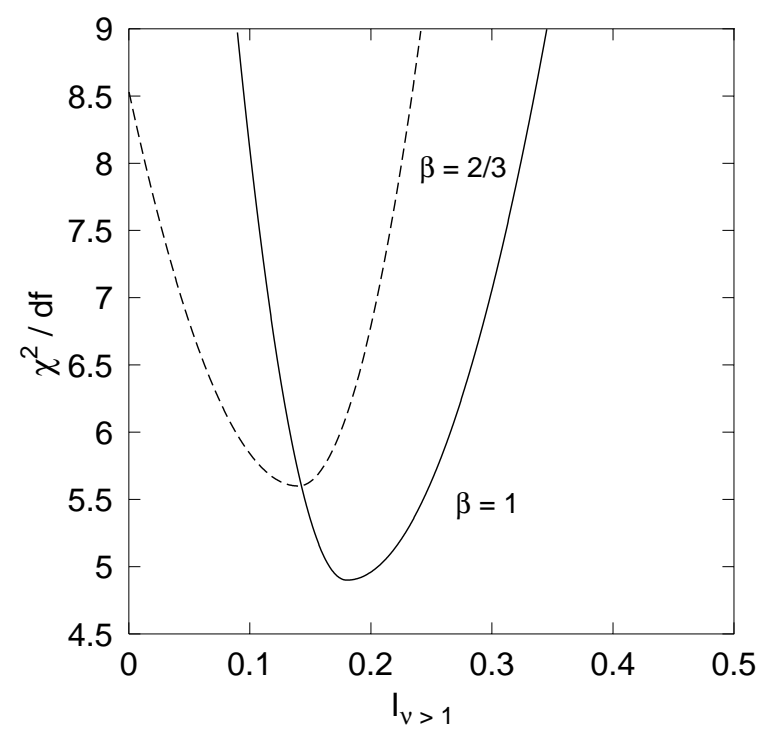

FIG. 7. Quality if fit, $\chi^{2}$ per degree of freedom, is plotted versus inelasticity of non-leading collisions, $I_{\nu>1}$, for our fits to $\mathrm{p}+$ nucleus data. Separate curves for the $\beta=2 / 3$ and $\beta=1$ cases are shown. The minima occur at $I_{\nu>1}=14 \%$ and $I_{\nu>1}=18 \%$, with the latter providing a somewhat better overall fit. 
TABLE I. Proton-proton and p-air inelastic cross sections with corresponding mean number of wounded nucleons from Eq. 8.

\begin{tabular}{|r|rr|r}
\hline \hline Energy $(\mathrm{GeV})$ & $\sigma_{p p}(\mathrm{mb})$ & $\sigma_{p-\text { air }}(\mathrm{mb})$ & $\langle\nu\rangle$ \\
\hline $10^{3}$ & 33.0 & 284. & 1.69 \\
$10^{7}$ & 67.0 & 427. & 2.29 \\
$10^{9}$ & 102. & 542. & 2.77 \\
$10^{11}$ & 142. & 661. & 3.14 \\
\hline \hline
\end{tabular}

TABLE II. Inelasticities for proton-proton interactions and for proton-air interactions for two classes of models: $\mathrm{A}=$ string-type models and $\mathrm{B}=$ statistical-type models (see text). The headings for $\mathrm{p}$-air correspond to different assumed values of $I_{\nu>1}$, e.g. $I_{\nu>1}=0.14,0.18$, etc.

\begin{tabular}{|r|cc|cccc|ccc|}
\hline \hline Energy $(\mathrm{GeV})$ & $\mathrm{pp}$ & $\left(I_{1}\right)$ & \multicolumn{5}{|c|}{ p-air $(\mathrm{A})$} & \multicolumn{2}{|c|}{ p-air (B) } \\
\hline $10^{3}$ & $\mathrm{~A}$ & $\mathrm{~B}$ & $I_{1}$ & .14 & .18 & $I(E)$ & .14 & .18 & $I_{1}$ \\
$10^{7}$ & .55 & .50 & .55 & .56 & .63 & .53 & .55 & .56 & .63 \\
$10^{9}$ & .57 & .19 & .62 & .64 & .74 & .58 & .38 & .40 & .45 \\
$10^{11}$ & .58 & .15 & .68 & .70 & .81 & .61 & .35 & .39 & .40 \\
\hline \hline
\end{tabular}

around $10^{18} \mathrm{eV}$. The model of Ref. 34 is an extrapolation to high energy of the work of Chou and Yang [35].

In the first group of models minimum-bias hadronic interactions proceed by the exchange of strings stretched between fragments of the incoming projectile and target particles. Strings radiate a characteristic multiplicity of secondaries per interval of rapidity, so the increase of multiciplicity is essentially logarithmic in energy (or more accurately, a power of the logarithm because the number of exchanged strings increases with energy). Inelasticity is determined basically by the momentum distributions of the valence constituents, increasing slightly with energy as more soft strings (coupled to sea quarks rather than valence quarks) are exchanged.

The cluster models are generally characterized by a more rapid, power-law dependence of multiplicity on invariant mass of the produced clusters. The observed rise of multiplicity in the central region is then matched by requiring the events to become increasingly elastic as energy increases, so that the fraction of total event energy going into particle production decreases while the fraction going into the leading nucleons increases. (See Ref. [36] for a discussion of inelasticity in the context of this class of models.)

In Table 1 we show estimates for pp and p-air cross sections along with the mean number of wounded nucleons per interaction from Eq. \& for lab energies ranging from ISR to those relevant in EAS analysis. We note here that the range of nuclei used in our study of inelasticity, Be $(A \approx 9)$ to $\mathrm{Pb}(A \approx 207)$ and $\mathrm{U}(A \approx 238)$, is nicely matched to the range of energies we wish to consider for hadron collisions in air. For a nucleus of mass 200, using a standard estimate of the p-nucleus cross section, 38 we find $\langle\nu\rangle_{200} \approx 3.77$ from Eq. 8 at low energy. In com- parison, the mean number of wounded nucleons expected in a proton-air collision at $10^{20} \mathrm{eV}$ from Table 1 is 3.14 .

Extrapolations of the two different types of models for hadron-hadron interactions beyond collider energies diverge significantly. We illustrate this in the first section of Table 2 by listing the inelasticity for $p p$ collisions, $I_{1}$, as a function of energy to represent the two classes of models. The $p p$ inelasticity in column $\mathrm{A}$ is chosen to be similar to that of Ref. [26], while that for B is from the work of Ding et al. 34. In both cases we use for illustration the traditional value of 0.5 at low energy, rather than the somewhat higher value implied by the distributions in Fig. 3.

Next we calculate the corresponding inelasticities for p-air collisions in the two classes of models starting from the the assumed values of $I_{1}$ and using Eqs. 4, 5, 6 and 7 to calculate overall inelasticity for various assumption about $I_{\nu>1}$. Based on the analysis of this paper, we use $I_{\nu>1}=0.14,0.18$. We also show the result of the 'naive' model of propagation through the nucleus $\left(I_{\nu>1}=I_{1}\right)$ for illustration, although we have seen that it is inconsistent with existing data. (The column labelled $\mathrm{I}(\mathrm{E})$ is discussed below.) At the highest energies, we see that 'statistical' models predict characteristic energy losses per collision of only 35 to $40 \%$ compared with about 60 to $70 \%$ for 'string' models. Even at energies characteristic of the "knee" region, the models are already predicting significant differences in energy deposition rates for proton initiated air showers.

The DPM and QGS models incorporate scattering on nuclear targets explicitly. For example, when only one target nucleon is wounded, a constituent quark(di-quark) belonging to the projectile proton couples to a string that in turn connects to a di-quark(quark) belonging to the wounded nucleon. In cases where there are two or more wounded nucleons in the target, the additional nucleons are coupled only to the sea quarks of the projectile. In this way the desired physics is reproduced by the model. In particular, the excited nucleon, being off mass-shell, does not interact repeatedly as a physical nucleon inside the nucleus. Moreover, the extra multiplicity characteristic of a collision on a nuclear target is naturally confined to the central region and the target fragmentation region of phase space. Capella et al. [27] point out that in their model the partial inelasticity $\left(I_{\nu>1}\right)$ is of order 0.2 , decreasing slightly on successive collisions in the same nucleus. We have checked that SIBYLL 26 also shows this behavior. Thus the string-type models are consistent with the result of our analysis of proton-proton and proton-nucleus collisions. In addition, we note that in this type of model it may be more natural to make the choice $\beta=1$; that is, to assume that the ultimate identity of the final state nucleon is determined only once during the interaction with the nucleus.

We note here that the singular nature of the sea quark distributions for small momentum fractions leads to a threshold effect in string-type models for $I_{\nu>1}$. Asymptotically the sea-quark momentum on the projectile side 


\section{SUMMARY}

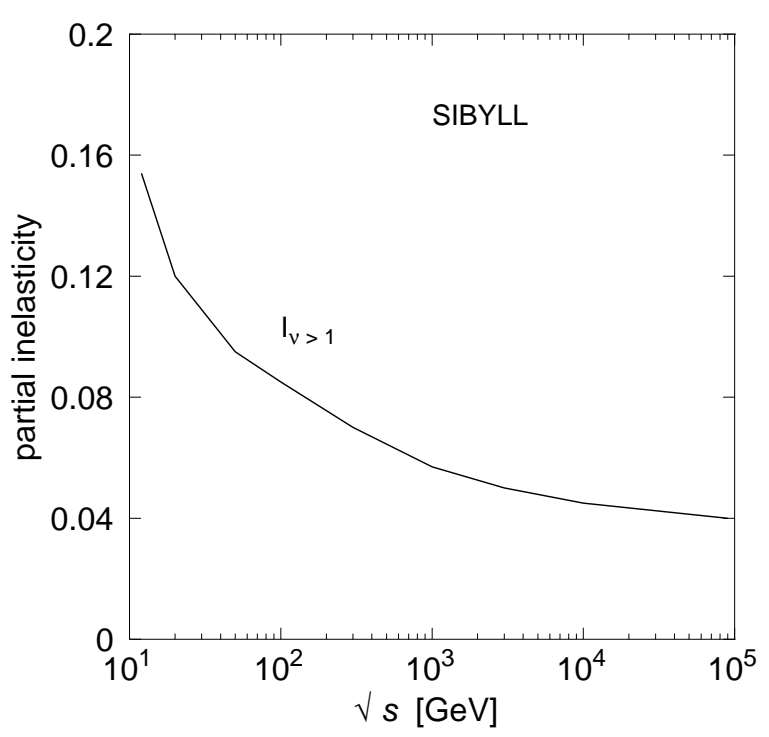

FIG. 8. Mean partial inelasticity, $I_{\nu>1}$, versus energy from the SIBYLL interaction model. The decrease with increasing energy is intrinsic to string-type models and is a consequence of the $x^{-1}$ singularity in the momentum distribution of sea quarks.

becomes negligible so that the fractional momentum removed from the projectile by wounded nucleons with $\nu>1$ is small at high energy. This leads to a decrease in the value of $I_{\nu>1}$ as energy increases. This behavior is characteristic of string-type interaction models. To illustrate, we use the joint probablility distribution for projectile partons from the SIBYLL interaction model [26] to evaluate the fraction of energy removed from the projectile for different numbers of wounded nucleons. Fig. (8) shows the result for $I_{\nu>1}$, averaged over different values of $\nu>1$. We see that energy losses due to interaction with the quark sea of the projectile decrease significantly at high energy. The column in Table 2 labeled $I(E)$ uses these energy dependent values of $I_{\nu>1}$ for the estimate of the overall inelasticity.

The generalization from $p p$ to $p$-nucleus is not prescribed in the statistical models, at least not in the form used by Ding et al. [34]. Given the observed rapid energy deposition in air showers, users of statistical models generally adopt the "naive" treatment of inelasticity in nuclei $\left(I_{\nu>1}=I_{1}\right)$ to compensate for the intrinsically high degree of elasticity of the hadron-hadron model. It has also been used in the context of some quark models of hadron-hadron interactions [37, making the hadronnucleus interactions highly inelastic.
Analysis of particle production in proton-proton and proton-nucleus collisions within a multiple scattering framework leads to the conclusion that the second and higher interactions of the excited nucleon inside the nucleus are relatively elastic. Assuming this feature of nuclear interactions persists to high energy, we can estimate the inelasticity in hadron-nucleus collisions beyond the energy range for which we have data. The results depend on the behavior of the cross section and inelasticity for proton-proton collisions, as illustrated in Tables 1 and 2. Since the column labelled $I_{\nu>1}=I_{1}$ is ruled out by the p-nucleus data, we conclude that the inelasticity on nuclear targets in the statistical models at high energy must be quite low. As pointed out in Ref. [34], (see also Ref. [39]), such low inelasticity is unable to account well for the Fly's Eye data. [40]39 Models of the type QGS and DPM represent interactions on nuclear targets in a way that is consistent with the low energy data on nuclear targets. They predict that inelasticity increases slowly with energy, with a modest increase for nuclear targets.

\section{ACKNOWLEDGMENTS}

This work was supported in part under Department of Energy Grant Number DE FG02 91 ER 40626.A007. 
[1] K. Greisen, Phys. Rev. Letters 16, 748 (1966); G.T. Zatsepin \& V.A. Kuz'min, JETP Letters 4, 78 (1966).

[2] A. Donnachie \& P.V. Landshoff, Phys. Lett. B296, 227 (1992). See also, "Review of Particle Physics", Phys. Rev. D54, 191 (1996).

[3] N. Hayashida et al., Phys. Rev. Letters 77, 1000 (1996); S. Yoshida et al., Astroparticle Physics 3, 105 (1993).

[4] "Proc. Int. Symposium on Extremely High Energy Csomic Rays: Astrophysics and Future Observatories" (ed. M. Nagano, Inst. for Cosmic Ray Research, Univ. of Tokyo) Sept. 1996. This volume includes descriptions of several new projects including the High Resolution Fly's Eye Detector, the Telescope Array Project and the Pierre Auger Project.

[5] "The Pierre Auger Project Design Report", Fermilab, November 1996.

[6] G.B. Khristiansen et al. (EAS-1000), Bull. Russ. Acad. Sci (Phys.) 58, 2002 (1994).

[7] Louis M. Barbier et al. (OWL Project, http://lheawww.gsfc.nasa.gov).

[8] A. Karle et al. (HEGRA Collaboration), Astroparticle Physics 3, 321 (1995).

[9] P. Doll et al. (KASCADE), Nucl. Phys. B (Proc. Suppl.) 14A, 336 (1990).

[10] M. Aglietta et al. (EASTOP-MACRO), Phys. Lett. B337, 376 (1994).

[11] M. Amenomori et al. (Tibet Array) Ap.J. 461, 408 (1996).

[12] T. Miller et al. (SPASE-AMANDA), Nucl. Phys. B (Proc. Suppl.) 43, 245 (1995).

[13] J. Knapp, D. Heck \& G. Schatz, Wissenschaftliche Berichte FZKA 5828 (1996).

[14] R. C. Hwa, Phys. Rev. Lett. 52, 492 (1984).

[15] J. Hufner and A. Klar, Phys. Lett. 145B, 167 (1984).

[16] R. J. Glauber and G. Matthiae, Nucl. Phys. B21, 135 (1970).

[17] J. Engel et al., Phys. Rev. D46, 5013 (1992).

[18] K. Goulianos, Phys. Rep. 101, 3 (1983).

[19] A. E. Brenner et al., Phys. Rev. D26, 1497 (1982).

[20] D. S. Barton et al., Phys. Rev. D27, 2580 (1983).

[21] J. Engler et al., Nucl. Phys. B84, 70 (1975).

[22] R. Bailey et al., Z. Phys. C29, 1 (1985).

[23] J. Ranft, Phys. Rev. D51, 64 (1995) and G. Battistoni, C. Forti \& J. Ranft, Astroparticle Physics 3, 157 (1995).

[24] K. Werner, Physics Reports 232 (1993) 87.

[25] N.N. Kalmykov \& S.S. Ostapchenko, Phys. At. Nucl. 56 (1993) 346.

[26] R.S. Fletcher, T.K. Gaisser, Paolo Lipari \& Todor Stanev, Phys. Rev. D50, 5710 (1994).

[27] A. Capella, U. Sukhatme, C.-I. Tan \& J. Tran Thanh Van, Physics Reports 236, 225 (1994).

[28] A.B. Kaidalov, K.A. Ter-Martirosyan \& Yu. M. Shabelsky, Yad. Fiz. 43 (1986) 1282.

[29] E. Fermi, Prog. Theor. Phys. (Kyoto) 5, 570 (1950).

[30] L.D. Landau in Men of Physics: L.D. Landau-II, ed. D. ter Haar, Pergamon, New York, 1969), pp. 131-151.

[31] J. Wdowczyk \& A.W. Wolfendale, J. Phys. G13, 411 (1987) and references therein.

[32] G.N. Fowler, A. Vourdas, R.M. Weiner \& G. Wilk, Phys. Rev. D35, 870 (1987) and references therein.

[33] R.M. Weiner, G. Wilk \& Z. Wlodarczyk, Phys. Rev. D45, 2308 (1992).

[34] L.K. Ding et al., Ap. J. 474, 490 (1997).

[35] T.T. Chou \& C.N. Yang, Phys. Rev. D32, 1692 (1985).

[36] Z. Wlodarczyk, inProc. XXIII Int. Cosmic Ray Conf. (ed. D.A. Leahy, R.B. Hicks \& D. Venkatesan, World Scientific, 1993) pp. 355-382.

[37] B.Z. Kopeliovich, N.N. Nikolaev \& I.K. Potashnikova, Phys. Rev. D39, 769 (1989).

[38] S.P. Denisov et al., Nucl. Phys. B61, 62 (1973).

[39] T.K. Gaisser et al., Phys. Rev. D47, 1919 (1993).

[40] D.J. Bird et al., Ap. J. 424, 491 (1994) and Phys. Rev. Letters 71, 3401 (1993). 